\title{
Effect of treadmill exercise combined with bone marrow stromal cell transplantation on atrophy-related signaling pathway in the denervated soleus muscle
}

\author{
Yeong-Hyun Cho, Tae-Beom Seo* \\ Department of Kinesiology, College of Natural Science, Jeju National University, Jeju, Korea
}

The purpose of this study was to investigate whether combination of low-intensity exercise with bone marrow stromal cell (BMSC) transplantation could regulate protein kinas B (Akt)- mammalian target of rapamycin (mTOR) and Wnt3a- $\beta$-catenin signaling pathways for prevention of soleus muscle atrophy after sciatic nerve injury (SNI). The experimental rats divided into 5 groups $(n=10)$ : normal control group, SNI+sedentary group (SED), SNI+low-intensity treadmill exercise group (TEX), SNI+BMSC transplantation group (BMSC), SNI+TEX+BMSC transplantation group (TEX+BMSC). Sciatic nerve crush injury was applied into the middle of thigh twice for $1 \mathrm{~min}$ and $30 \mathrm{sec}$ at interval. Low-intensity treadmill exercise was comprised of walking at a speed of 4 to $8 \mathrm{~m} / \mathrm{min}$ for 30 min once a day. cultured BMSC at a density of $5 \times 10^{6}$ in $50-\mu \mathrm{L}$ phosphate-buffered saline was injected into the distal portion of the injured sciatic nerves. TEX+BMSC group dramatically upregulated expression levels of growth-associated protein-43 in the in- jured sciatic nerve at 2 weeks postinjury. Also, although Akt and mTOR signaling pathway significantly increased in TEX and BMSC groups than SED group, TEX+BMSC group showed more potent increment on this signaling in soleus muscle after SNI. Lastly, Wnt3a and the nuclear translocation of $\beta$-catenin and nuclear factor-kappa $B$ in soleus were increased by SNI, but TEX+BMSC group significantly downregulated activity of this signaling pathway in the nuclear cell lysate of soleus muscle. Present findings provide new information that combination of low-intensity treadmill exercise might be effective therapeutic approach on restriction of soleus muscle atrophy after peripheral nerve injury.

Keywords: Sciatic nerve, Treadmill exercise, Bone marrow stromal cell transplantation, Wnt3a

\section{INTRODUCTION}

It has been known that peripheral nerve injury, including the sciatic nerve, adversely affects quality of life due to prolonged neuropathic pain and motor dysfunction (Zhang et al., 2021). The movement of the human body is closely associated with synaptic plasticity in the central and peripheral nervous systems as well as the release of neurotransmitter (acetylcholine) in the neuromuscular junction (NMJ) of the skeletal muscle fibers. Sciatic nerve innervated the skeletal muscles in the lower leg is the largest peripheral nerve in the human body (Sladjana et al., 2008). Therefore, it has been known that traumatic sciatic nerve injury (SNI) resulted in locomotor dysfunction through muscle atrophy in the foot and thigh (Ganguly et al., 2017), and these functional problems are quite limited in their recovery (Irintchev, 2011).

From a molecular biological point of view, SNI-induced muscle atrophy is caused by inhibition of protein kinas B (Akt)-mammalian target of rapamycin (mTOR) signaling pathway and promoting of the Wnt3a- $\beta$-catenin cascades (Kurimoto et al., 2015). mTOR has been known as one of the most widely recognized major proteins for regulating muscle hypertrophy (Yoon, 2017), promoting mTOR signaling cascade by activating Akt (Nandagopal and Roux, 2015). Also, Wnt plays a critical role in various development stages ranging from cell survival and apoptosis (Clevers and Nusse, 2012; Kurimoto et al., 2015), which is classified as noncanonical ( $\beta$-catenin independent) and canonical ( $\beta$-catenin
${ }^{*}$ Corresponding author: Tae-Beom Seo (D) https://orcid.org/0000-0001-9213-2251 Department of Kinesiology, College of Natural Science, Jeju National University, 102 Jejudaehak-ro, Jeju 63243, Korea

Email: seotb@jejunu.ac.kr

Received: October 28, 2021 / Accepted: November 9, 2021
This is an Open Access article distributed under the terms of the Creative Commons Attribution Non-Commercial License (https://creativecommons.org/licenses/by-nc/4.0/) which permits unrestricted non-commercial use, distribution, and reproduction in any medium, provided the original work is properly cited. 
dependent) signaling pathway (Albanese et al., 2017). Canonical Wnts is a general metabolic regulator to activate the nuclear translocation of $\beta$-catenin (Li et al., 2012) and interactively affect nuclear factor-kappa B (NF-kB). Some previous studies reported that inhibition of Wnt3a-regulated nuclear $\beta$-catenin accumulation might support activation of acetylcholine receptors $(\mathrm{AChR})$ in NMJ of skeletal muscle (Wang et al., 2008) and NF-kB activated by canonical Wnt pathway might delay development of skeletal myogenesis in patients with muscle disorders (Bakkar and Guttridge, 2010). However, other previous studies suggested conflicting results that voluntary wheel running (Cheng et al., 2013) or treadmill running exercise (Aschenbach et al., 2006) significantly increased canonical Wnt- $\beta$-catenin pathway to prevent the muscle atrophy. Thus, therapeutic potential of Wnt signaling pathway in the skeletal muscle and nervous system remain poorly understood and still controversial.

To accelerate axonal regeneration and functional recovery after SNI, many researchers have widely applied cell transplantation techniques using bone marrow stromal cell (BMSC), neural stem cell, fibroblasts (Ma et al., 2014; Zhao et al., 2020), and they suggested that engrafting BMSC was most effective in promoting Schwann cell proliferation and axonal regrowth in the injured sciatic nerve without side effects (Mohammadi et al., 2012; Wang et al., 2020). In addition, forced or voluntary exercise is one of the rehabilitation approaches to delay muscle atrophy and improve functional recovery after SNI (Boeltz et al., 2013). Looking at some previous studies that investigated the effect of aerobic exercise on skeletal muscle morphology in sciatic nerve regeneration, it was represented that both low-intensity forced and voluntary exercises apparently increased myofibril cross-sectional areas and less collagen around soleus muscle fibers after SNI (Bonetti et al., 2017) as well as facilitated hypertrophy in innervated soleus and gastrocnemius muscles after SNI via increase of regenerating motor axons and activation of Akt-mTOR signaling pathway (Jaiswal et al., 2020; Yuan et al., 2020).

With these results presented by previous studies, BMSC transplantation or low-intensity aerobic exercise is a positive effector for improving locomotor functions after SNI. However, experiment studies on combined application of treadmill exercise and BMSC transplantation to examine cellular and molecular mechanisms of muscle atrophy after SNI are still lacking. Therefore, the purpose of this study was to investigate whether combined low-intensity treadmill training with BMSC engraftment could regulate hypertrophy- and atrophy-related signaling pathway in the soleus after SNI.

\section{MATERIALS AND METHODS}

\section{Experimental animals}

Male Sprague-Dawley rats (7 weeks old) were purchased for this experiment. The use of rats in this study was approved by Ethical committee of Jeju National University (approval number: 20190026). And experimental procedures were conducted in accordance with the guidelines for the Care and Use of Laboratory Animals at Jeju National University. The animals in vivo experiment were divided into five groups with randomization method: the normal control group (CON, $n=10), S N I+$ sedentary group (SED, $n=10)$, SNI+low-intensity treadmill exercise group (TEX, $n=10)$, SNI+ BMSC transplantation group (BMSC, $\mathrm{n}=10$ ), $\mathrm{SNI}+\mathrm{TEX}+\mathrm{BMSC}$ transplantation group (TEX+BMSC, $n=10)$. Animals were maintained at a constant room temperature of $22^{\circ} \mathrm{C}$ and $60 \%$ of humidity under 12/12-hr light-dark cycle. They were accepted to eat commercial rat chow (Samyang Co., Seoul, Korea) and water ad libitum.

\section{BMSC culture}

BMSCs were collected from femur and tibia of young rats ( 4 weeks old), as the extraction technique described by Kim et al. (2018) and Nakano et al. (2013). Cells were cultured in Dulbecco's modified Eagle's medium with $20 \%$ fetal calf serum. Five to 7 days later, BMSCs adhered to the base on the culture dishes proliferated to a density of ca. $5 \times 10^{6}$ in one dish.

\section{SNI and BMSC transplantation}

All experiment animals used anesthetized with using an animal inhalation narcosis control (Jeungdo Bio \& Plant, Seoul, Korea). First, the rats were placed into the chamber with $2 \%-2.5 \%$ concentration of isoflurane for anesthesia and then 1.5\%-1.8\% concentration for maintenance during SNI. Sciatic nerve crush injury was applied into the middle of thigh twice for $1 \mathrm{~min}$ and $30 \mathrm{sec}$ at interval (Seo et al., 2006). Single dose of $5 \times 10^{6}$ harvested BMSCs in $30 \mu \mathrm{L}$ phosphate-buffered saline (PBS) was injected into the injury area using a 30-gauge needle. After surgery, anesthetized animals were then placed on a heating pad maintained at $37^{\circ} \mathrm{C}$, and then they were put in their cages for resting.

\section{Treadmill exercise}

All rats in this experiment had a treadmill exercise adaptation period for 2 weeks. All animals in exercise groups were rested for 2 days after SNI, and started low-intensity treadmill exercise on third postoperation day. Low-intensity exercise on the treadmill 
device (Jeungdo Bio \& Plant) was comprised of walking at a speed of 4 to $8 \mathrm{~m} / \mathrm{min}$ for 30 min once a day for 2 weeks postinjury.

\section{Western blot analysis}

Protein lysates were extracted from the dissected sciatic nerve tissues into triton lysis buffer, and the nucleus and cytoplasm were separated by nuclear extraction buffer and cytosol extraction buffer. Denatured proteins were separated on sodium dodecyl sulphate-polyacrylamide gel and then transferred onto polyvinylidene difluoride membrane on ice at $200 \mathrm{~mA}$ for $2 \mathrm{hr}$. The membranes were blocked with $5 \%$ skim milk, $0.1 \%$ Tween 20 in tris buffered saline for $30 \mathrm{~min}$ at room temperature. Then, the membranes were incubated overnight with primary antibodies at $4^{\circ} \mathrm{C}$. Protein $(20 \mu \mathrm{g})$ was used for Western blot analysis using anti-GAP-43 mouse monoclonal antibody (1:1,000, Santa Cruz Biotechnology, Santa Cruz, CA, USA), anti-phosphorylated mTOR rabbit polyclonal antibody (1:1,000, Cell Signaling Biotechnology, Danvers, MA, USA), anti-phosphorylated Akt rabbit polyclonal antibody (1:1,000, Cell Signaling Biotechnology), anti-Wnt3a rabbit monoclonal antibody (1:1,000, Cell Signaling Biotechnology), anti- $\beta$ catenin mouse monoclonal antibody $(1: 1,000$, Santa Cruz Biotechnology), anti-NF-kB rabbit polyclonal antibody (1:1,000, Cell Signaling Biotechnology), anti- $\beta$-actin mouse monoclonal antibody (1:2,000, Santa Cruz Biotechnology), anti-GAPDH rabbit polyclonal antibody (1:1,000, Cell Signaling Biotechnology), and goat anti-mouse or goat anti-rabbit horseradish peroxidase-conjugated secondary antibody (1:1,000, GeneTex Inc., Irvine, CA, USA) were used. The blotting proteins were detected by using Westar ECL substrates (Cyanagen, Bologna, Italy). Analysis of protein density was performed using Chemidoc (Bio-Rad, Hercules, CA, USA).

\section{Immunofluorescence staining}

For detection of sprouting axons in the injured sciatic nerve, tissues were embedded and frozen at $20^{\circ} \mathrm{C}$. Cross sections $(20 \mu \mathrm{m})$ were cut on a cryostat. Sections were fixed with $4 \%$ paraformaldehyde and $4 \%$ sucrose in PBS at room temperature for $40 \mathrm{~min}$, permeabilized with $0.5 \%$ Nonidet P-40 in PBS, and blocked with $2.5 \%$ horse serum and $2.5 \%$ bovine serum albumin for $4 \mathrm{hr}$ at room temperature. The sections were incubated with anti-neurofilament-200 (NF-200) rabbit polyclonal antibody (1:700) (SigmaAldrich, St. Louis, MO, USA). And then, they were incubated with rhodamine-goat anti-rabbit secondary antibody (1:600) (Molecular Probes, Eugene, OR, USA) for $1 \mathrm{~h}$ at room temperature. The stained samples were viewed with a fluorescence microscope (Nikon model E-600, Nikon, Kawasaki, Japan), and the images were cap- tured with a digital camera, and analyzed using Adobe Photoshop Software (version CS6, San Jose, CA, USA). The number and regenerating axons were evaluated by using i-Solution software (Image and Microscope Technology, Goleta, CA, USA).

\section{Statistical analysis}

All the data is presented as a mean \pm standard error. Statistical analysis was performed using one-way analysis of variane followed by Duncan post hoc test. The significance level was set at $P<0.05$. All graphs were performed by using Prism 6 (GraphPad, La Jolla, CA, USA).

\section{RESULTS}

\section{Expression levels of GAP-43 in the injured sciatic nerve}

To confirm the effect of combined low-intensity exercise with BMSC transplantation on expression levels of GAP-43, specific regeneration marker, in the sciatic nerve at 2 weeks after SNI, we performed biochemical and histological analysis. As shown in Fig. 1A, GAP-43 levels were significantly increased in TEX and TEX+ BMSC groups compared to those in SED group. In immunofluorescence staining at 2 weeks after SNI, the number of NF-200stained axons was higher in TEX, BMSC, and TEX+BMSC groups than those seen in the SED group (Fig. 1B).

\section{Activation of Akt/mTOR signaling pathway in the soleus after SNI}

To examine activation of Akt-mTOR signaling pathway in the soleus at 2 weeks after SNI, we performed Western blot analysis using anti-Akt and mTOR antibodies. As shown in Fig. 2, expression levels of phosphorylated Akt and mTOR were significantly upregulated in TEX or BMSC group compared to those in SED group. In particular, TEX+BMSC group showed more potent increment on phosphorylated Akt and mTOR levels in the soleus compared to TEX group.

\section{Activation of canonical Wnt3a- $\beta$-catenin signaling pathway in soleus after SNI}

To examine activation of the nuclear translocation of $\beta$-catenin in soleus after SNI, we analyzed induction levels of $\beta$-catenin from the whole cell or nuclear cell lysate. As shown in Fig. 3, in whole cell lysate, Wnt3a was significantly increased in SED group compared to those in CON group, but TEX group dramatically downregulated Wnt3a levels. In particular, TEX+BMSC group showed more potent decrement on Wnt3a levels in the soleus compared 

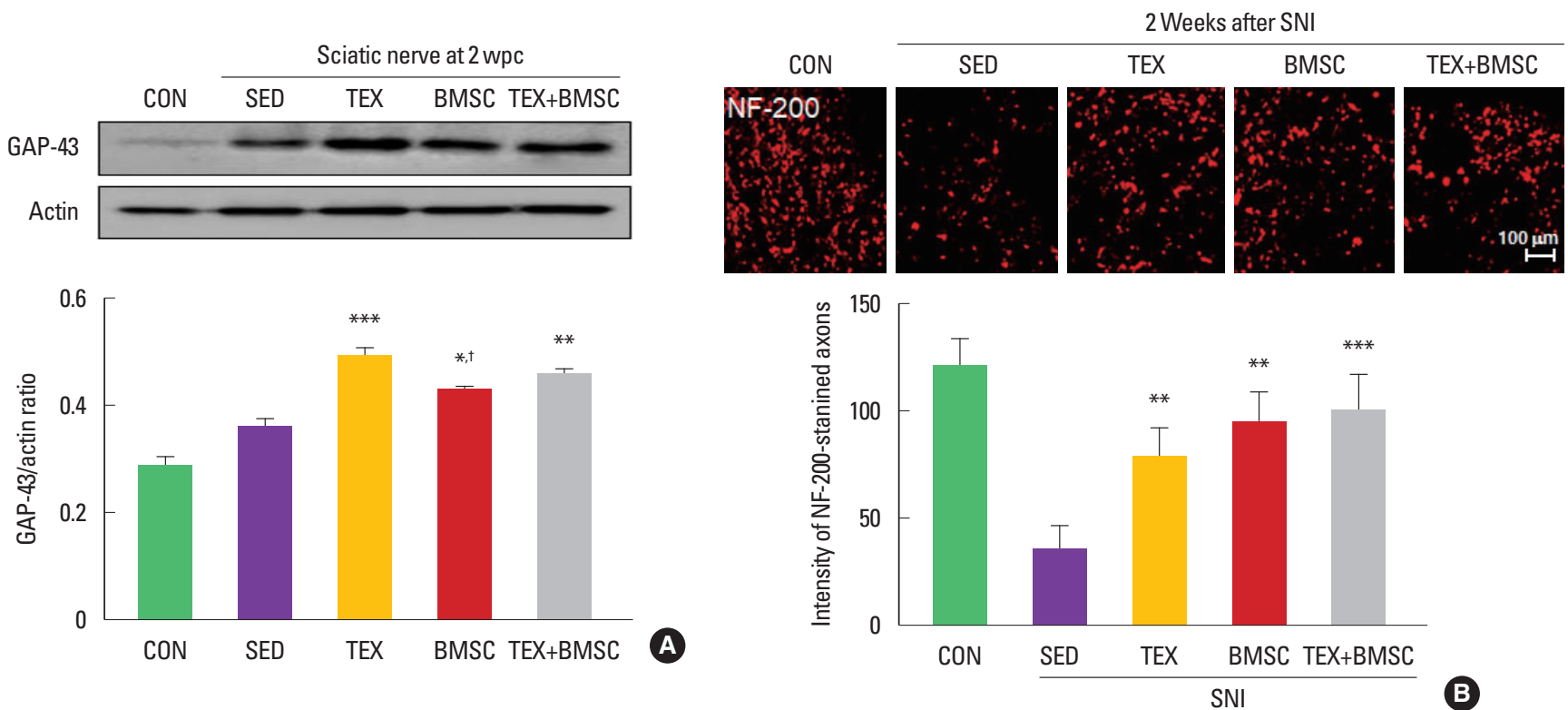

Fig. 1. Combination of low-intensity treadmill exercise with bone marrow stromal cell (BMSC) transplantation regulates expression levels of growth-associated protein-43 (GAP-43) and number of neurofilament-200 (NF-200)-stained axons after sciatic nerve injury (SNI). (A) TEX and TEX+BMSC groups significantly increased GAP43 induction levels in whole cell lysate of the sciatic nerve 2 weeks after SNI. Upper panel: representative expression of GAP-43 protein. Lower panel: the quantification of the ratio of GAP-43 to actin. (B) To investigate regenerating axons in the injured sciatic nerve, immunofluorescence staining was applied into $10 \mathrm{~mm}$ distal region to the injury site 2 weeks after SNI. TEX, BMSC, and TEX+BMSC groups showed a significant increase in the number of NF-200-stained regenerating axons when compared to other groups. Upper panel: immunofluorescence staining of NF-200 antibody. Lower panel: the quantification of the intensity of NF-200-positive axons. wpc, week post crush; CON, normal control group; SED, SNI+sedentary group; TEX, SNI+low-intensity treadmill exercise group; BMSC, SNI+BMSC transplantation group; TEX+BMSC, SNI+TEX+BMSC transplantation group. ${ }^{*} P<0.05,{ }^{*} P<0.01,{ }^{* * *} P<0.001$ vs. CON group. ${ }^{\dagger} P<0.05$ vs. TEX group
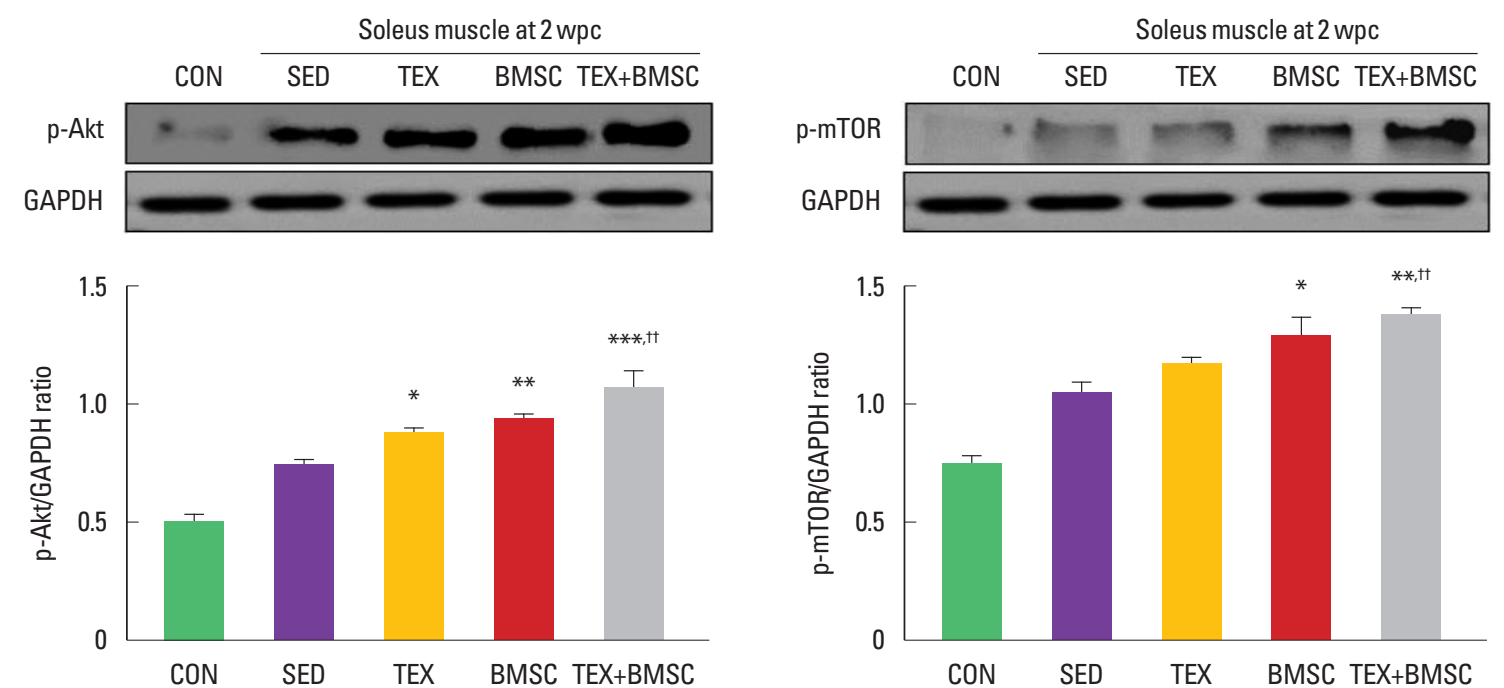

Fig. 2. Combination of low-intensity treadmill exercise with bone marrow stromal cell (BMSC) transplantation regulates muscle hypertrophy-related signaling pathway after sciatic nerve injury (SNI). Phosphorylated-protein kinase B (p-Akt) protein was significantly increase in soleus muscle of TEX, BMSC, and TEX+BMSC groups. Phosphorylated-mammalian target of rapamycin (p-mTOR) was further enhanced in BMSC and TEX+BMSC groups than SED group. In particular, both p-Akt and mTOR were dramatically upregulated in TEX+BMSC group. Upper panel: representative expression of p-Akt and mTOR proteins. Lower left panel: the quantification of the ratio of p-Akt to actin. Lower right panel: the quantification of the ratio of p-mTOR to actin. wpc, week post crush; CON, normal control; SED, SNI+sedentary group; TEX, SNI+low-intensity treadmill exercise group; BMSC, SNI+BMSC transplantation group; TEX+BMSC, SNI+TEX+BMSC transplantation group. ${ }^{*} P<0.05$, ${ }^{* *} P<0.01,{ }^{* * *} P<0.001$ vs. CON group. ${ }^{\dagger \dagger} P<0.01$ vs. TEX group. 

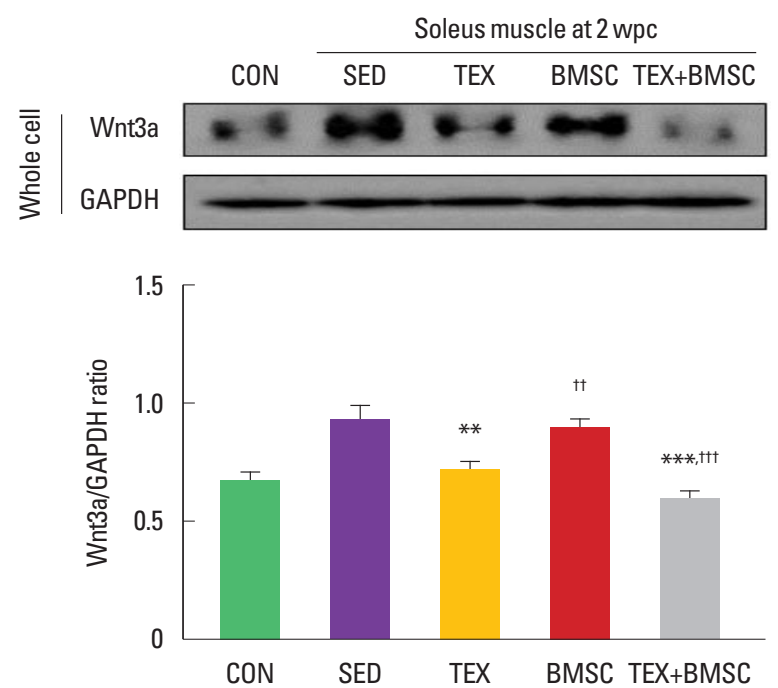
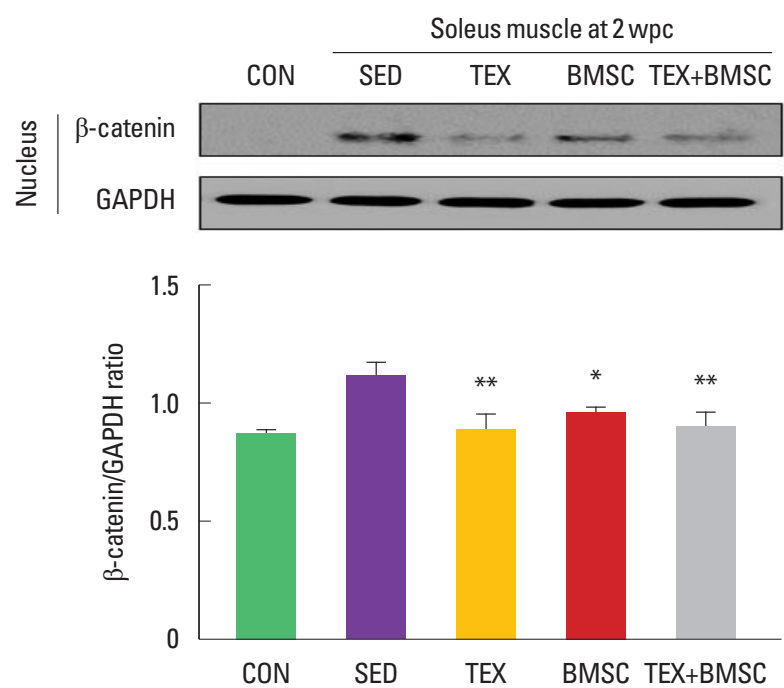

Fig. 3. Combination of low-intensity treadmill exercise with bone marrow stromal cell (BMSC) transplantation regulates canonical Wnt3a- $\beta$-catenin signaling pathway in soleus muscle after sciatic nerve injury (SNI). In whole cell lysate, Wnt3a was more decreased in soleus of TEX and TEX+BMSC groups than SED group, but applying combination of treadmill exercise and BMSC transplantation sharply downregulated Wnt3a expression levels in soleus compared to TEX group. In nuclear cell lysate, translocation of $\beta$-catenin to the nucleus of soleus muscle after SNI was significantly decreased in TEX, BMSC, and TEX+BMSC groups. Upper left panel: representative expression of Wnt3a in whole cell lysate. Upper right panel: representative expression of $\beta$-catenin in nuclear cell lysate. Lower left panel: the quantification of the ratio of Wnt3a to actin. Lower right panel: the quantification of the ratio of $\beta$-catenin to actin. wpc, week post crush; CON, normal control; SED, SNI+ sedentary group; TEX, SNI+low-intensity treadmill exercise group; BMSC, SNI+BMSC transplantation group; TEX+BMSC, SNI+TEX+BMSC transplantation group. ${ }^{*} P<0.05,{ }^{* *} P<0.01,{ }^{* *} P<0.001$ vs. CON group. ${ }^{t \dagger} P<0.01,{ }^{\text {tt }} P<0.001$ vs. TEX group.

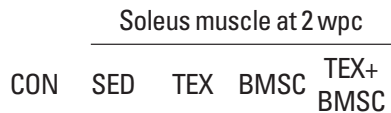
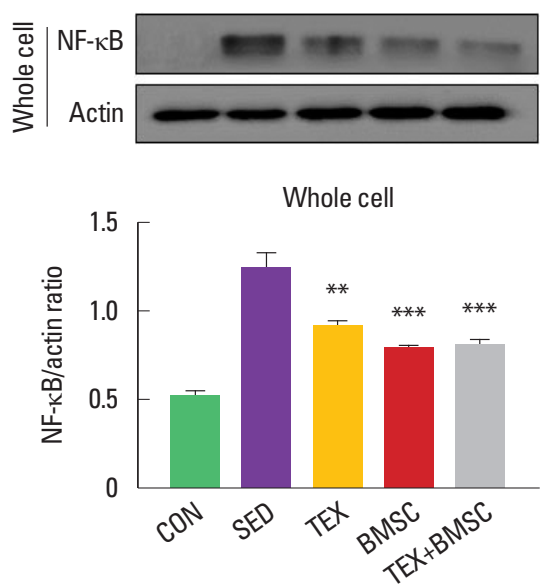
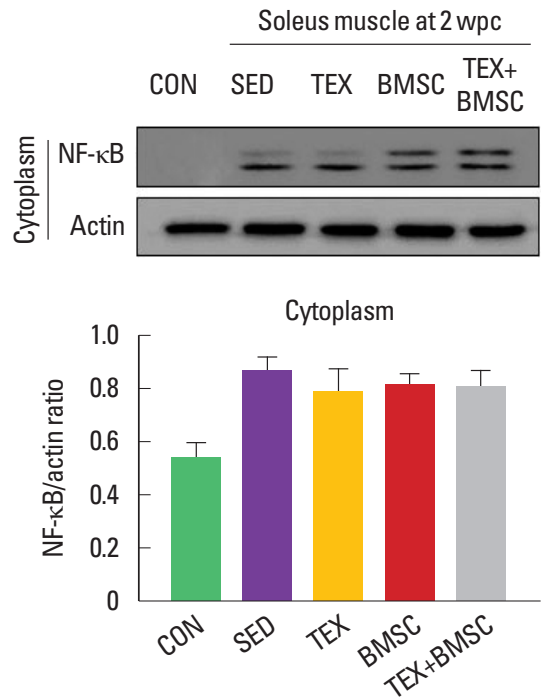
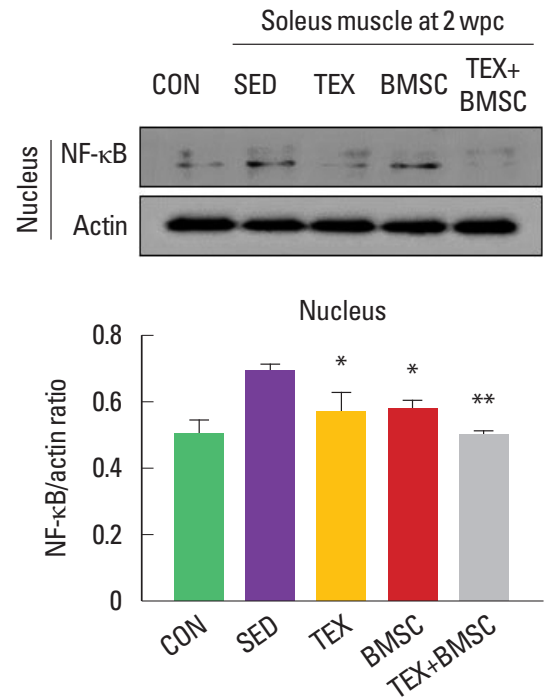

Fig. 4. Combination of low-intensity treadmill exercise with bone marrow stromal cell (BMSC) transplantation regulates expression of nuclear factor-kappa B (NF- $\mathrm{KB}$ ), a specific inflammation marker, in soleus muscle after sciatic nerve injury (SNI). In whole cell lysate, NF-kB was more increased in soleus of SED group than CON

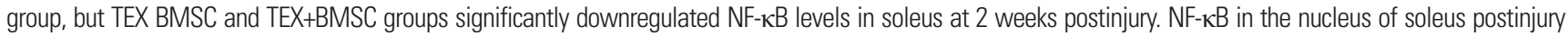
was significantly decreased in TEX and BMSC. In particular, TEX+BMSC groups showed the greatest downregulation of NF- $\mathrm{kB}$ in both whole and nuclear cell lysates.

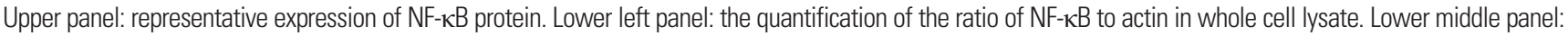

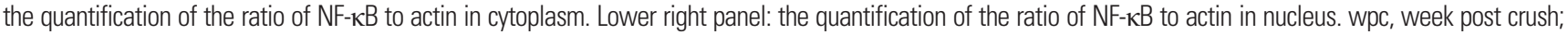
CON, normal control; SED, SNI+sedentary group; TEX, SNI+low-intensity treadmill exercise group; BMSC, SNI+BMSC transplantation group; TEX+BMSC, SNI+TEX+ BMSC transplantation group. ${ }^{*} P<0.05,{ }^{* *} P<0.01,{ }^{* * *} P<0.001$ vs. CON group. 
to TEX group. Activation of the nuclear translocation of $\beta$-catenin in soleus was significantly decreased in TEX, BMSC, and TEX+ BMSC groups.

\section{Expression levels of NF-KB in soleus after SNI}

$\mathrm{NF}-\mathrm{kB}$ is a key molecule of inflammation during development and tissue regeneration. To confirm translocation of NF- $\mathrm{KB}$ to the nucleus in soleus muscle after $\mathrm{SNI}$, we investigated alterations of $\mathrm{NF}-\kappa \mathrm{B}$ from the whole cell or nuclear cell lysate. As shown in Fig. 4, in whole cell lysate, NF- $\mathrm{kB}$ was significantly enhanced in SED group compared to those in other groups, but TEX, BMSC, and TEX+BMSC groups dramatically downregulated NF- $\mathrm{kB}$ levels. But, there were no significant differences of NF- $\mathrm{B}$ expression levels in the cytoplasm between all experimental groups. The nuclear NF-kB accumulation was enhanced in SED group and TEX, BMSC, and TEX+BMSC groups significantly decreased translocation of NF- $\mathrm{kB}$ into the nucleus compared to SED group.

\section{DISCUSSION}

Various previous studies that applied regular exercise and cell transplantation for sciatic nerve regeneration mainly focused on axonal regrowth in the nerves and pain improvement in dorsal root ganglions, but these results do not provide an appropriate mechanism on exercise-induced skeletal muscle changes for functional recovery after SNI. Therefore, we tried to find the effective molecular mechanisms to suppress atrophy of soleus muscle after SNI.

The injured peripheral nerves undergo Wallerian degeneration, which is morphological and biochemical changes in the injured axons. Regeneration process includes Schwann cell proliferation, migration, and differentiation as well as axonal sprouting and remyelination around the injured areas (Caillaud et al., 2019). Among the various regeneration-related molecules, GAP- 43 has been well known as a crucial marker secreted from the axon terminal of the injured peripheral nerves (Chung et al., 2020). Jiang et al. (2017) suggested that GAP- 43 could promote the axonal growth and remyelination for functional recovery after sciatic nerve transection. Thus, we first confirmed expression levels of GAP-43 in the injured sciatic nerve using Western blot analysis. TEX and TEX+ BMSC groups significantly increased GAP-43 levels compared to SED group, but not in BMSC group. In addition, there are no significant differences TEX and TEX+BMSC groups. In previous studies, GAP-43 started to be expressed from the early phase of the sciatic nerve regeneration and treadmill exercise continuously increased from 1 day to 2 weeks after SNI (Kim et al., 2020; Seo et al., 2009). BMSC-dervied extracellular vesicles treatment also promoted the functional recovery and nerve regeneration after SNI through upregulation of GAP-43 protein (Ma et al., 2017). Although the results in group treated with only BMSC are slightly different from previous data, it is thought that there is a synergistic effect when looking at the findings of combined application of exercise and BMSC.

SNI leads to skeletal muscle atrophy and functional dysfunction (Bonetti et al., 2017). Atrophy of the skeletal muscle is highly associated with a specific mechanism such as phosphorylation of mTOR and canonical Wnt signaling pathway (Bodine et al., 2001; Kurimoto et al., 2015; Nandagopal and Roux, 2015). mTOR regulates protein synthesis and muscle growth, and it is stimulated by combining resistance exercise and appropriate nutritional strategies (Deldicque et al., 2005). Insulin-like growth factor 1, PtdIns3-OH kinase, and PtdIns-regulated kinase Akt led to activation of mTOR, whose downstream molecules including P70 ${ }^{\text {s6k }}$ (protein 70 kD ribosomal protein S6 kinase) and 4E-BP1 (eukaryotic translation initiation factor $4 \mathrm{E}$ binding protein 1) have promoted protein synthesis and muscle hypertrophy through increases in the initiation and elongation phases of translation (Bodine et al., 2001; Nandagopal and Roux, 2015). Unlike noncanonical Wnt pathway, activation of canonical Wnt signaling pathway accumulate and translocate $\beta$-catenin into the nucleus for reducing the sensitivity of AChR in NMJ and skeletal muscle mass (Albanese et al., 2017; Zhang and Peng, 2011). In present study, TEX+BMSC group showed the greatest increase in the activation of Akt-mTOR signaling pathway as well as the greatest decrease in translocation of $\beta$-catenin into the nucleus of soleus muscle after SNI. Previous studies demonstrated that activation of Akt/mTOR pathway could increase Schwann cell proliferation and decrease skeletal muscle atrophy after peripheral nerve injury (Zhu et al., 2019) and physical immobilization (Bodine et al., 2001), respectively. Although canonical Wnt/ $\beta$-catenin signaling pathway is controversial in the remodeling of damaged aged skeletal muscles, many studies have reported that this pathway is generally activated during muscle atrophy and suppressed by physical exercise (Hu et al., 2019; Zhang et al., 2021). These data implicate that combination of low-intensity exercise with BMSC transplantation might be a new therapeutic strategy for improving skeletal muscle atrophy after SNI.

In addition to elucidating the role of canonical Wnt/ $\beta$-catenin signaling pathway in muscle atrophy, we identified that TEX+ BMSC group sharply downregulated NF- $\kappa \mathrm{B}$ levels in soleus at 2 weeks postinjury compared to those in other groups. Skeletal 
muscle atrophy can result from enhanced protein degradation and downregulated protein synthesis in immobilization (Bar-Shai et al., 2005), aging (Tilstra et al., 2011), and chronic inflammatory diseases (Li et al., 2008) through activation of NF-kB. In disuse muscle atrophy such as sciatic denervation, NF- $\kappa \mathrm{B}$ can regulate expression of myostatin, p38, and calcineurin to induce the loss of skeletal muscle mass (Zhang et al., 2007), and this signaling pathway reciprocally influences the activity of canonical Wnt/ $\beta$-catenin signaling pathway (Ma and Hottiger, 2016). Our results regarding NF- $\mathrm{kB}$ secretion blocked by exercise was consistent with some previous showing that NF- $\kappa \mathrm{B}$ and Wnt pathways shows multiple cross-talks during skeletal muscle atrophy (Ma and Hottiger, 2016).

Given these results obtained in present study, low-intensity treadmill exercise and BMSC transplantation approaches would positively regulate biochemical mechanisms in nerve and soleus muscle during sciatic nerve regeneration. In conclusion, our findings suggest that combination of low-intensity treadmill exercise with BMSC transplantation should provide may be effective therapeutic approach to restrict soleus muscle atrophy after peripheral nerve injury.

\section{CONFLICT OF INTEREST}

No potential conflict of interest relevant to this article was reported.

\section{ACKNOWLEDGMENTS}

This work was supported by the National Research Foundation of Korea (NRF) grant funded by the Korea government (MSIT) (No. 2019R1F1A1062392).

\section{REFERENCES}

Albanese I, Yu B, Al-Kindi H, Barratt B, Ott L, Al-Refai M, de Varennes B, Shum-Tim D, Cerruti M, Gourgas O, Rhéaume E, Tardif JC, Schwertani A. Role of noncanonical Wnt signaling pathway in human aortic valve calcification. Arterioscler Thromb Vasc Biol 2017;37:543-552.

Aschenbach WG, Ho RC, Sakamoto K, Fujii N, Li Y, Kim YB, Hirshman

MF, Goodyear LJ. Regulation of dishevelled and $\beta$-catenin in rat skel-

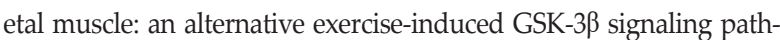
way. Am J Physiol Endocrinol Metab 2006;291:E152-E158.

Bakkar N, Guttridge DC. NF-kappaB signaling: a tale of two pathways in skeletal myogenesis. Physiol Rev 2010;90:495-511.

Bar-Shai M, Carmeli E, Reznick AZ. The role of NF- $\kappa \mathrm{B}$ in protein break- down in immobilization, aging, and exercise: from basic processes to promotion of health. Ann N Y Acad Sci 2005;1057:431-447.

Bodine SC, Stitt TN, Gonzalez M, Kline WO, Stover GL, Bauerlein R, Zlotchenko E, Scrimgeour A, Lawrence JC, Glass DJ, Yancopoulos GD. Akt/mTOR pathway is a crucial regulator of skeletal muscle hypertrophy and can prevent muscle atrophy in vivo. Nat Cell Biol 2001;3: 1014-1019.

Boeltz T, Ireland M, Mathis K, Nicolini J, Poplavski K, Rose SJ, Wilson E, English AW. Effects of treadmill training on functional recovery following peripheral nerve injury in rats. J Neurophysiol 2013;109:26452657.

Bonetti LV, Malysz T, Ilha J, Barbosa S, Achaval M, Faccioni-Heuser MC. The effects of two different exercise programs on the ultrastructural features of the sciatic nerve and soleus muscle after sciatic crush. Anat Rec (Hoboken) 2017;300:1654-1661.

Caillaud M, Richard L, Vallat JM, Desmoulière A, Billet F. Peripheral nerve regeneration and intraneural revascularization. Neural Regen Res 2019; 14:24-33.

Cheng A, Morsch M, Murata Y, Ghazanfari N, Reddel SW, Phillips WD. Sequence of age-associated changes to the mouse neuromuscular junction and the protective effects of voluntary exercise. PLoS One 2013;8: e67970.

Chung D, Shum A, Caraveo G. GAP-43 and BASP1 in axon regeneration: Implications for the treatment of neurodegenerative diseases. Front Cell Dev Biol 2020;8:567537.

Clevers H, Nusse R. Wnt/ $\beta$-catenin signaling and disease. Cell 2012;149: 1192-1205.

Deldicque L, Theisen D, Francaux M. Regulation of mTOR by amino acids and resistance exercise in skeletal muscle. Eur J Appl Physiol 2005; 94:1-10.

Ganguly A, McEwen C, Troy EL, Colburn RW, Caggiano AO, Schallert TJ, Parry TJ. Recovery of sensorimotor function following sciatic nerve injury across multiple rat strains. J Neurosci Methods 2017;275:25-32.

Hu J, Shi D, Ding M, Huang T, Gu R, Xiao J, Xian CJ, Dong J, Wang L, Liao $\mathrm{H}$. Calmodulin-dependent signalling pathways are activated and mediate the acute inflammatory response of injured skeletal muscle. J Physiol 2019;597:5161-5177.

Irintchev A. Potentials and limitations of peripheral nerve injury models in rodents with particular reference to the femoral nerve. Ann Anat 2011;193:276-285.

Jaiswal PB, Tung JK, Gross RE, English AW. Motoneuron activity is required for enhancements in functional recovery after peripheral nerve injury in exercised female mice. J Neurosci Res 2020;98:448-457.

Jiang B, Zhang Y, Zhao J, She C, Zhou X, Dong Q, Wang P. Effects of localized $X$-ray irradiation on peripheral nerve regeneration in transect- 
ed sciatic nerve in rats. Radiat Res 2017;188:455-462.

Kim JE, Cho YH, Seo TB. Treadmill exercise activates ATF3 and ERK1/2 downstream molecules to facilitate axonal regrowth after sciatic nerve injury. J Exerc Rehabil 2020;16:141-147.

Kim SH, Cho JH, Lee YH, Lee JH, Kim SS, Kim MY, Lee MG, Kang WY, Lee KS, Ahn YK, Jeong MH, Kim HS. Improvement in left ventricular function with intracoronary mesenchymal stem cell therapy in a patient with anterior wall ST-segment elevation myocardial infarction. Cardiovasc Drugs Ther 2018;32:329-338.

Kurimoto S, Jung J, Tapadia M, Lengfeld J, Agalliu D, Waterman M, Mozaffar T, Gupta R. Activation of the Wnt/ $\beta$-catenin signaling cascade after traumatic nerve injury. Neuroscience 2015;294:101-108.

Li W, Khor TO, Xu C, Shen G, Jeong WS, Yu S, Kong AN. Activation of Nrf2-antioxidant signaling attenuates NFkappaB-inflammatory response and elicits apoptosis. Biochem Pharmacol 2008;76:1485-1489.

Li Y, Shao Y, Tong Y, Shen T, Zhang J, Li Y, Gu H, Li F. Nucleo-cytoplasmic shuttling of PAK4 modulates $\beta$-catenin intracellular translocation and signaling. Biochim Biophys Acta 2012;1823:465-475.

Ma B, Hottiger MO. Crosstalk between Wnt/ $\beta$-catenin and NF- $\kappa B$ signaling pathway during Inflammation. Front Immunol 2016;7:378.

Ma F, Xiao Z, Chen B, Hou X, Dai J, Xu R. Linear ordered collagen scaffolds loaded with collagen-binding basic fibroblast growth factor facilitate recovery of sciatic nerve injury in rats. Tissue Eng Part A 2014; 20:1253-1262.

Ma Y, Ge S, Zhang J, Zhou D, Li L, Wang X, Su J. Mesenchymal stem cellderived extracellular vesicles promote nerve regeneration after sciatic nerve crush injury in rats. Int J Clin Exp Pathol 2017;10:10032-10039.

Mohammadi R, Azizi S, Delirezh N, Hobbenaghi R, Amini K, Malekkhetabi $P$. The use of undifferentiated bone marrow stromal cells for sciatic nerve regeneration in rats. Int J Oral Maxillofac Surg 2012;41:650-656.

Nakano N, Nakai Y, Seo TB, Homma T, Yamada Y, Ohta M, Suzuki Y, Nakatani T, Fukushima M, Hayashibe M, Ide C. Effects of bone marrow stromal cell transplantation through CSF on the subacute and chronic spinal cord injury in rats. PLoS One 2013;8:e73494.

Nandagopal N, Roux PP. Regulation of global and specific mRNA translation by the mTOR signaling pathway. Translation (Austin) 2015;3: e983402.

Seo TB, Han IS, Yoon JH, Seol IC, Kim YS, Jo HK, An JJ, Hong KE, Seo YB, Kim DH, Park SK, Yang DC, Namgung U. Growth-promoting activity of Hominis Placenta extract on regenerating sciatic nerve. Acta Phar- macol Sin 2006;27:50-58

Seo TB, Oh MJ, You BG, Kwon KB, Chang IA, Yoon JH, Lee CY, Namgung $\mathrm{U}$. ERK1/2-mediated Schwann cell proliferation in the regenerating sciatic nerve by treadmill training. J Neurotrauma 2009;26:1733-1744.

Sladjana UZ, Ivan JD, Bratislav SD. Microanatomical structure of the human sciatic nerve. Surg Radiol Anat 2008;30:619-626.

Tilstra JS, Clauson CL, Niedernhofer LJ, Robbins PD. NF-kB in aging and disease. Aging Dis 2011;2:449-465.

Wang H, Jia Y, Li J, Liu Q. Schwann cell derived exosomes induce bone marrow derived mesenchymal stem cells to express Schwann cell markers in vitro. Mol Med Rep 2020;21:1640-1646.

Wang J, Ruan NJ, Qian L, Lei WL, Chen F, Luo ZG. Wnt/B-catenin signaling suppresses Rapsyn expression and inhibits acetylcholine receptor clustering at the neuromuscular junction. J Biol Chem 2008;283: 21668-21675.

Yoon MS. mTOR as a key regulator in maintaining skeletal muscle mass. Front Physiol 2017;8:788.

Yuan Y, Li D, Yu F, Kang X, Xu H, Zhang P. Effects of Akt/mTOR/p70S6K signaling pathway regulation on neuron remodeling caused by translocation repair. Front Neurosci 2020;14:565870.

Zhang HL, Peng HB. Mechanism of acetylcholine receptor cluster formation induced by DC electric field. PLoS One 2011;6:e26805.

Zhang Y, Zhao D, Li X, Gao B, Sun C, Zhou S, Ma Y, Chen X, Xu D. The Wnt/ $\beta$-catenin pathway regulated cytokines for pathological neuropathic pain in chronic compression of dorsal root ganglion model. Neural Plast 2021;2021:6680192.

Zhang Z, Reenstra W, Weiner DJ, Louboutin JP, Wilson JM. The p38 mitogen-activated protein kinase signaling pathway is coupled to Tolllike receptor 5 to mediate gene regulation in response to Pseudomonas aeruginosa infection in human airway epithelial cells. Infect Immun 2007;75:5985-5992.

Zhao J, Ding Y, He R, Huang K, Liu L, Jiang C, Liu Z, Wang Y, Yan X, Cao F, Huang X, Peng Y, Ren R, He Y, Cui T, Zhang Q, Zhang X, Liu Q, Li Y, Ma Z, Yi X. Dose-effect relationship and molecular mechanism by which BMSC-derived exosomes promote peripheral nerve regeneration after crush injury. Stem Cell Res Ther 2020;11:360.

Zhu S, Tian Z, Torigoe D, Zhao J, Xie P, Sugizaki T, Sato M, Horiguchi H, Terada K, Kadomatsu T, Miyata K, Oike Y. Aging- and obesity-related peri-muscular adipose tissue accelerates muscle atrophy. PLoS One 2019;14:e221366. 